Walter P. Lysenko

Daniel P. Rusthoi

K.C. Dominic Chan

G. H. Gillespie

B. W. Hill
AOT-1

AOT-1

AOT-9

G.H. Gillespie

G.H. Gillespie Associates

RECEIVED

OCT 301996

Q.STI

1996 Computational Accelerator Physics Conference

Williamsburg, VA

September 24-27, 1996
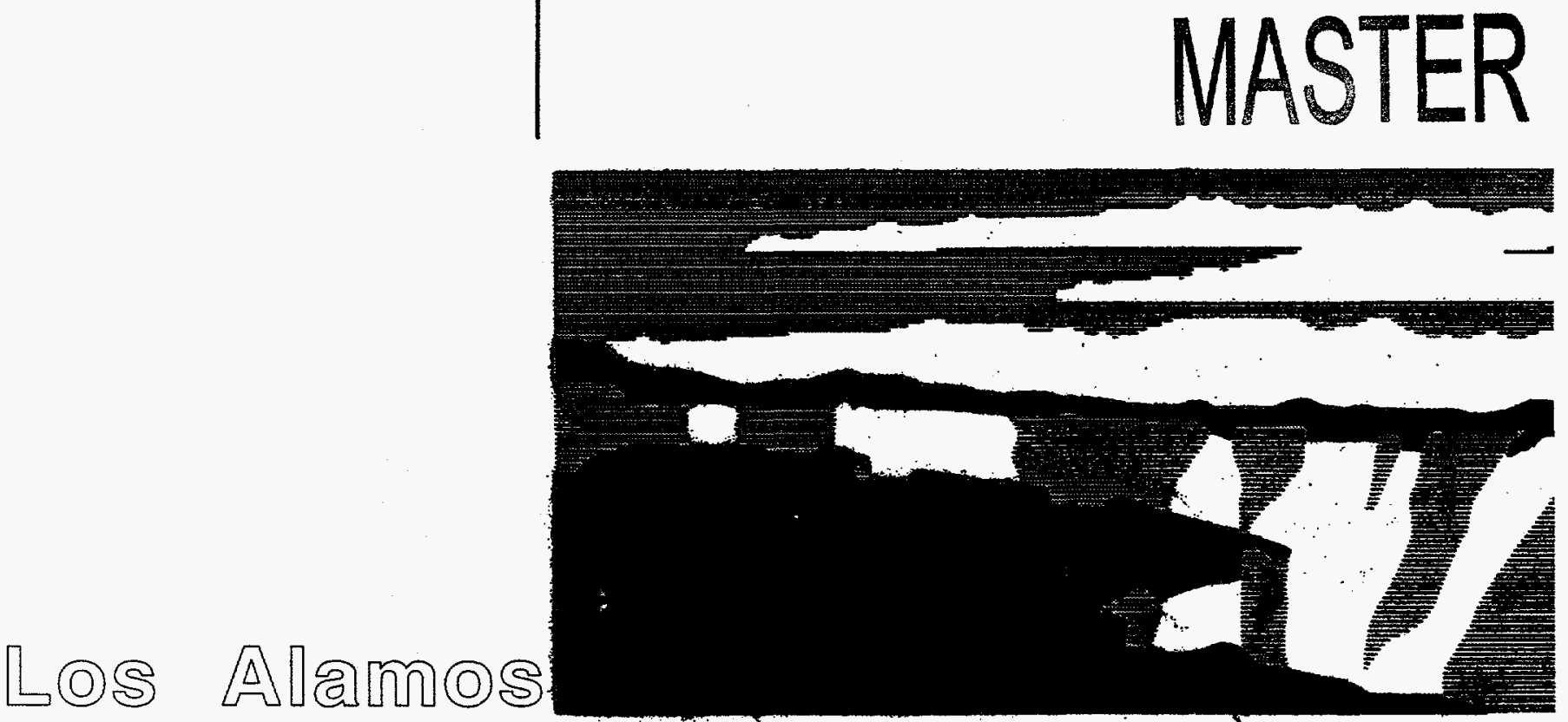

NATIONAL

L A BORATOR Y

Los Alamos National Laboratory, an affirmative action/equal opportunity employer, is operated by the University of California for the U.S. Department of Energy under contract W-7405-ENG-36. By acceptance of this article, the publisher recognizes that the U.S. Government retains a nonexclusive, royalty-free license to publish or reproduce the published form of this contribution, or to allow others to do so, for U.S. Govemment purposes. The Los Alamos National Laboratory requests that the publisher identify this article as work performed under the auspices of the U.S. Department of Energy. 


\section{DISCLAIMER}

This report was prepared as an account of work sponsored by an agency of the United States Government. Neither the United States Government nor any agency thereof, nor any of their employees, makes any warranty, express or implied, or assumes any legal liability or responsibility for the accuracy, completeness, or usefulness of any information, apparatus, product, or process disclosed, or represents that its use would not infringe privately owned rights. Reference herein to any specific commercial product, process, or service by trade name, trademark, manufacturer, or otherwise does not necessarily constitute or imply its endorsement, recommendation, or favoring by the United States Government or any agency thereof. The views and opinions of authors expressed herein do not necessarily state or reflect those of the United States Government or any agency thereof. 


\section{DISCLAIMER}

Portions of this document may be illegible in electronic image products. Images are produced from the best available original document. 


\title{
TRACE 3-D Code Improvements
}

\author{
W. P. Lysenko, D. P. Rusthoi, K. C. D. Chan, \\ AOT-1, MS H808, Los Alamos National Lab., Los Alamos, NM 87545, USA \\ G. H. Gillespie and B. W. Hill \\ G. H. Gillespie Associates, Inc., P.O. Box 2961, Del Mar, CA 92014, USA
}

\begin{abstract}
TRACE 3-D is an interactive beam-transport code for bunched beams that includes accelerating elements and linear space-charge forces. It has been integrated with an improved GUI (graphic user interface) based on the Shell for Particle Accelerator Related Codes. Recent modifications to the code include centroid tracking and an improved beam description consisting of a set of beam slices, each having its own $6 \mathrm{D}$ centroid and sigma matrix. This allows us to study some nonlinear effects, such as wakefields, that are related to the variation of the beam bunch along the longitudinal direction.
\end{abstract}

\section{INTRODUCTION}

TRACE 3-D (1) is an interactive beam-transport code for bunched beams. It includes accelerating elements and linear space-charge forces. It is useful for designing linear accelerators and transport lines because of its ease of use and its ability to do matching and other types of optimizations. TRACE 3 -D describes a beam by a $6 \times 6$ sigma matrix, which is the collection of second moments of the phase-space distribution. Since the code computes the evolution of the distribution directly instead of dealing with the single-particle motion, it is a natural environment for matching computations.

Recently, we added two new capabilities to TRACE 3-D. We now track centroids in addition to sigma matrices. This allows us to study offsets in beams and optical elements. Also, in a more substantial change, we can now represent the beam bunch as a collection of slices that interact with the external forces and each other. This allows us to study certain nonlinear effects like short-time (single bunch) wakefields. The changes to the code were made in manner that was very compatible with the existing code structure, existing input data files, and the GUI (graphic user interface) of the MacTrace ${ }^{\mathrm{TM}}$ version $(5,6)$. Bunch slicing is turned on by setting the existing variable IBS, which controls the generation of the initial beam, to a value of 2 . 
Simulation of wakefield effects has been reviewed by Chan (2). Our approach follows that of the LTRACK code (3), which was originated by Chao and Cooper (4). What is new here is that we do not assume that all the particles have velocity $c$. Our code can take into account, for example, bunch lengthening caused by space-charge interactions between the bunch slices.

\section{CENTROIDS}

Initial beam offsets are specified in a new array in the data file. Element offsets are introduced by a new rotate/translate element that replaces the old rotate element. Any offset automatically turns on centroid tracking. Centroids are plotted on the usual graphs (profiles and phase-space). The quadrupole element also has been expanded to include built-in rotations and offsets that can take on random values. The centroid capability is useful for aperture studies and is also required for the wakefield feature, described next.

\section{BUNCH SLICING AND WAKEFIELDS}

When we use the new wakefield feature, the beam bunch is divided into a user-defined number of slices longitudinally. We describe each slice by its $6 \mathrm{D}$ centroid and its $6 \times 6$ sigma matrix. The code generates this collection of slices from the usual input Courant-Snyder parameters and emittances.

\section{Generating the Initial Beam .}

We assume a uniformly filled, upright ellipsoid in $(x, y, z)$ with $-z_{\max } \leq$ $z \leq+z_{\max }$. We make $2 N+1$ equal-length slices, labeled from $-N$ (head) to $+N$ (tail). Slice number 0 is centered at $z=0$. Let $z_{i}$ be the $z$ value at the upstream face of slice $i$ and define $z_{N+1}$ to be $-z_{\max }$. The number of particles in a slice of length $d z$ at position $z$ is proportional to $\left(1-\left(z / z_{\max }\right)^{2}\right) d z$. With this distribution, the $z$-centroid of slice $i$ is given by

$$
<z>_{i}=\frac{6 z_{i}^{2}-3 z_{i}^{4} / z_{\max }^{2}-6 z_{i+1}^{2}+3 z_{i+1}^{4} / z_{\max }^{2}}{12 z_{i}-4 z_{i}^{3} / z_{\max }^{2}-12 z_{i+1}+4 z_{i+1}^{3} / z_{\max }^{2}}
$$

and the fraction of particles in slice $i$ is given by

$$
N_{i}=\frac{1}{4}\left(3 \frac{z_{i}}{z_{\max }}-\left(\frac{z_{i}}{z_{\max }}\right)^{3}-3 \frac{z_{i+1}}{z_{\max }}+\left(\frac{z_{i+1}}{z_{\max }}\right)^{3}\right) .
$$

To estimate the $z^{\prime}$ centroids $\left\langle z^{\prime}\right\rangle_{i}$, we assume the ratio $\left\langle z^{\prime}\right\rangle_{i} \mid\langle z\rangle_{i}$ is the same as $z_{e}^{\prime} / z_{m}$, where $z_{m}$ is the maximum value of $z$ for the $z-z^{\prime}$ ellipse and $z_{e}^{\prime}$ is the value of $z^{\prime}$ at $z=z_{m}$. Since $z_{m}=\sqrt{\beta_{z} \epsilon_{z}}$ and $z_{e}^{\prime}=-\alpha_{z} \sqrt{\epsilon_{z} / \beta_{z}}$, we have

$$
<z^{\prime}>_{i}=-\frac{\alpha_{z}}{\beta_{z}}<z>_{i}
$$


for the value of the $z^{\prime}$ centroid of slice $i$. When we compute the slice sigma matrices, we adjust the $x$ and $y$ emittances at each slice according to

$$
\epsilon_{x, y} \rightarrow\left[1-\left(\frac{\left\langle z>_{i}\right.}{z_{\max }}\right)^{2}\right] \frac{\epsilon_{x, y}}{f},
$$

where the factor $f$ is the ratio of average to maximum emittance value

$$
f=\sum_{i=-N}^{N}\left[1-\left(\frac{<z>_{i}}{z_{\max }}\right)^{2}\right] N_{i}
$$

The $\sigma_{55}$ and $\sigma_{66}$ for the $i$-th slice are estimated from

$$
\begin{gathered}
\sigma_{55 i}=(\Delta z / 2)^{2}, \\
\sigma_{66 i}=\left[\frac{\epsilon_{z}}{\beta_{z}}-\left(\frac{\left\langle z>_{i}\right.}{\beta_{z}}\right)^{2}\right] \frac{1}{e},
\end{gathered}
$$

where $e$ is the ratio of average to maximum $\sigma_{66}$ value

$$
e=\frac{\beta_{z}}{\epsilon_{z}} \sum_{-N}^{N}\left[\frac{\epsilon_{z}}{\beta_{z}}-\left(\frac{<z>_{i}}{\beta_{z}}\right)^{2}\right]
$$

\section{Recombining the Slices}

Whenever the codes needs to generate output or compute space charge, we combine the slices into a single centroid and sigma matrix. Then we use existing mechanisms in the code to generate output or compute space charge. Let $u$ represent $\left(x, x^{\prime}, y, y^{\prime}, z, z^{\prime}\right)$. The overall centroid components are given by

$$
<u>=\sum_{i=-N}^{N} N_{i}<u>_{i}
$$

The matrix elements $\sigma_{i j}$ for the overall sigma matrix are defined by

$$
\left.\sigma_{i j} / 5=<\left(u_{i}-<u_{i}>\right)\left(u_{j}-<u_{j}>\right)>=<u_{i} u_{j}>-<u_{i}><u_{j}\right\rangle .
$$

The matrix elements for the $k$-th slice are given by

$$
\sigma_{i j k} / 5=\left\langle u_{i} u_{j}\right\rangle_{k}-\left\langle u_{i}\right\rangle_{k}\left\langle u_{j}\right\rangle_{k} .
$$

The overall sigma matrix can be determined to be

$$
\frac{\sigma_{i j}}{5}=\sum_{k=-N}^{N} N_{k}\left(\frac{\sigma_{i j k}}{5}+\left\langle u_{i}\right\rangle\left\langle u_{j}\right\rangle\right)-\left(\sum_{k=-N}^{N} N_{k}<u_{i}>_{k}\right)\left(\sum_{k=-N}^{N} N_{k}<u_{j}>_{k}\right) \text {. }
$$




\section{Transporting the Beam}

The bunch centroid is transported as a single particle using the $R$ matrix at each step in the simulation. Space charge does not contribute to this motion. For a sliced-beam simulation, the slice centroids are transported similarly except that they are also acted upon by space charge forces, as described later.

In a sliced-beam simulation, the sigma matrix for each slice is transported similarly to the bunch sigma matrix in the original code. The new wakefield elements have a new feature, however, in that each slice has a different $R$ matrix that acts on it, which depends on the slices ahead.

\section{Space Charge}

When we compute space charge in a wakefield run, we combine the slice centroids and sigma matrices into an overall sigma matrix, which we use to compute the space-charge $R$ matrix. This $R$ matrix then transports all the slice sigma matrices.

The overall centroid of the beam is not affected by space charge. For a wakefield simulation, however, we have a collection of slice centroids and these are affected by space charge. To handle this, we subtract the overall centroid from the slice centroids to get a collection of relative centroids. We then transport these using the space-charge $R$ matrix.

The overall sigma matrix is checked for correlations to determine if the spatial ellipsoid is tilted, just as in the usual no-wakefield situation. If there is a correlation (this can be caused by a bend, rotation, or wakefield), we rotate into a coordinate system in which the overall spatial ellipsoid is upright.

\section{Wakefield Elements}

Three new elements have been added to TRACE 3-D to model the monopole, dipole, and quadrupole wakefields. These elements are inserted immediately following the element that is responsible for generating the wakefield. The monopole wakefield changes the energies of the bunch slices, the dipole wakefield cause deflections of the transverse centroids of the slices, while the quadrupole wakefield affects the sigma matrices of the slices in addition to the energy and transverse centroids. The three wakefield multipoles are expressed in terms of a wake function strength per unit length, $W_{o}(s), W_{1}(s)$, and $W_{2}(s)$. Each wakefield acts over the length $L$ of the element responsible for generating the wakefield. The wakefield functions are modeled as second degree polynomials:

$$
\begin{aligned}
& L W_{o}(s)=p_{o}(1)+p_{o}(2) s+p_{o}(3) s^{2} \\
& L W_{1}(s)=p_{1}(1)+p_{1}(2) s+p_{1}(3) s^{2}
\end{aligned}
$$


TABLE I. Comparison of output beams for test problem with no wakefields.

\begin{tabular}{lccccccccc} 
Mode & $\alpha_{x}$ & $\beta_{x}$ & $\epsilon_{x}$ & $\alpha_{y}$ & $\beta_{y}$ & $\epsilon_{y}$ & $\alpha_{z}$ & $\beta_{z}$ & $\epsilon_{z}$ \\
\hline IBS=0 (no slicing) & 4.49 & 1.28 & 6.00 & 10.9 & 1.72 & 6.00 & 33.6 & 47.3 & 40.0 \\
IBS=2 (with slicing) & 4.49 & 1.28 & 6.00 & 10.9 & 1.72 & 6.00 & 33.1 & 46.5 & 39.8 \\
IBS=0 (0 mA) & 3.51 & .955 & 6.00 & 5.70 & .844 & 6.00 & 15.5 & 22.7 & 40.0 \\
\hline
\end{tabular}

$$
L W_{2}(s)=p_{2}(1)+p_{2}(2) s+p_{2}(3) s^{2}
$$

The three coefficients for a given multipole, $p_{m}(1), p_{m}(2)$, and $p_{m}(3)$ are user inputs for the wakefield elements. Slice centroids and sigma matrices are transformed by the wakefield elements as in the LTRACK code (3).

\section{INTEGRATION WITH THE GUI}

The changes to TRACE 3-D described here have integrated into an improved version of the GUI based on the Shell for Particle Accelerator Codes, which is used in the MacTrace ${ }^{\text {TM }}$ implementation of TRACE 3-D $(5,6)$. Of particular importance is the user defined element facility, which is how the new wakefield elements have been implemented. The TableBuilder application in the GUI allows the user to create custom Piece Windows, which are used to graphically construct beamlines containing the user-defined elements.

\section{TEST RESULTS}

Table I compares three TRACE 3- D runs for the same beamline and initial beam. There are no wakefields. The first line shows a normal run (no slicing). The second line shows a five-slice wakefield run with zero wakefields. The third line shows a normal no-slicing run with space charge turned off. We see that space charge causes a significant bunch lengthening (increase in $\beta_{z}$ ) and that the bunch-slicing run correctly simulates this behavior. Generally, bunch slicing is valid whenever the longitudinal temperature is not too high. This representation of the distribution breaks down if the velocity spread is large enough to cause the slices to overlap significantly during the simulation.

Figure 1 shows part of the graphical output of a TRACE 3-D run with a quadrupole wakefield. Phase-space ellipses are shown for all five slices. Transverse ellipses near the tail are rotated relative to those near the head. This accounts for the emittance growth. The wakefield function for this example case peaks at about $1.5 \mathrm{~mm}$, which is approximately the bunch length. 

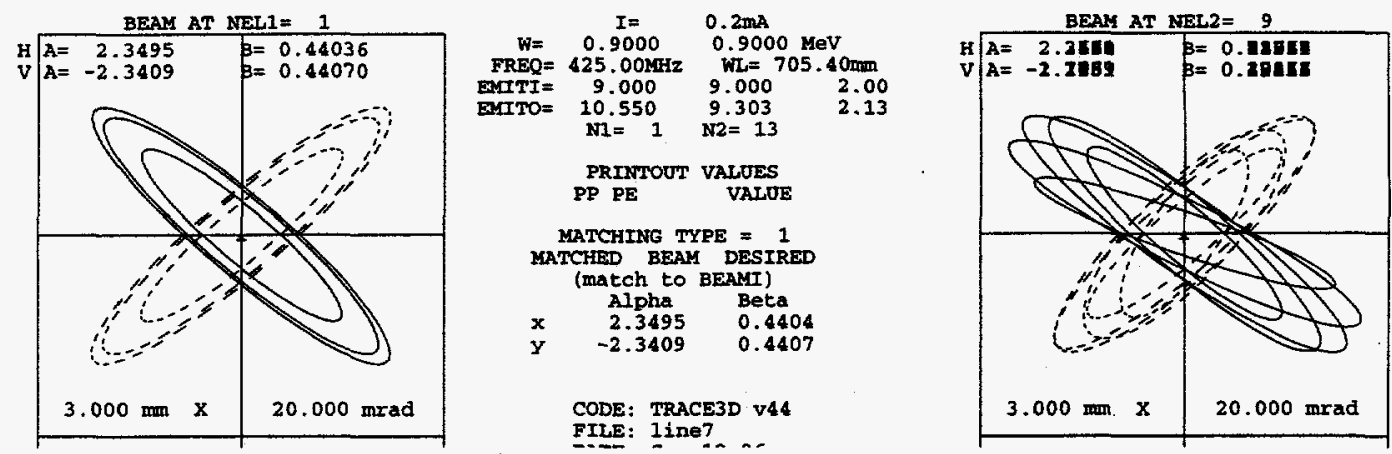

FIGURE 1. Quadrupole wakefield example. Phase-space ellipses for each slice are shown. Initial beam is at left, final is at right. Solid curves are for $x$, dashed are for $y$.

\section{CONCLUSION}

We have demonstrated the flexibility in the moment approach in computational beam dynamics by introducing a significant new capability to the TRACE 3-D code. This is especially significant because of the ease of use of this code, particularly in the MacTrace ${ }^{\mathrm{TM}}$ implementation.

\section{REFERENCES}

1. Crandall, K. and Rusthoi, D., TRACE 3-D Documentation, Los Alamos National Laboratory Report LA-UR-90-4146, 1990.

2. Chan, K. C. D., "Computer Codes for Wakefield Analysis in rf-Based FreeElectron Laser," in Proceedings of the Beijing FEL Seminar, Singapore, World Scientific Publishing Co. Pte. Ltd., 1989, pp.172-192.

3. Chan, K. C. D. and Cooper, R. C., "LTRACK-Beam-Transport Calculation Including Wakefield Effects," in AIP Conference Proceedings 177, New York, American Institute of Physics, 1988, p.37-44.

4. Chao, A. W. and Cooper, R. K., "Transverse Quadrupole Wake Field Effects in High Intensity Linacs," Particle Accelerators 13, 1-12, (1983).

5. Gillespie, G. H., "The Shell for Particle Accelerator Related Codes (SPARC) A Unique Graphical User Interface," in AIP Conference Proceedings 297, New York, American Institute of Physics, 1993, pp.576-583.

6. Gillespie, G. H. and Hill, B. W., "A Graphical User Interface for TRACE 3-D Incorporating Some Expert System Type Features," in 1992 Linear Accelerator Conference Proceedings (Ottawa), AECL-10728, 1992, pp. 787-789. 